MODELING, IDENTIFICATION AND CONTROL, 2000, vOL. 21, NO. 4, 241-253

doi: $10.4173 /$ mic.2000.4.3

\title{
Catalyst Deactivation: Control Relevance of Model Assumptions
}

\author{
BERNT LIE† and DAVID M. HIMMELBLAU $\ddagger$
}

\begin{abstract}
Keywords: Catalyst deactivation, dynamic models, population balance, activity
\end{abstract} model, analysis

Two principles for describing catalyst deactivation are discussed, one based on the deactivation mechanism, the other based on the activity and catalyst age distribution. When the model is based upon activity decay, it is common to use a mean activity developed from the steady-state residence time distribution. We compare control-relevant properties of such an approach with those of a model based upon the deactivation mechanism. Using a continuous stirred tank reactor as an example, we show that the mechanistic approach and the population balance approach lead to identical models. However, common additional assumptions used for activitybased models lead to model properties that may deviate considerably from the correct one.

\section{Introduction}

The loss of activity is an important problem in catalysis (Fogler, 1992). Many mechanisms have been proposed to explain the observed deactivation (Butt and Petersen, 1988). Fogler (1992) divides catalyst deactivation into three categories: deactivation by sintering, deactivation by coking or fouling, and deactivation by poisoning. Levenspiel (1972) gives a similar classification of deactivation mechanisms. Other mechanisms that occur in commercial reactors are, e.g., deactivation by volatilization or leaching, deactivation by thermal excursions, and changes in catalyst oxidation state.

The two main principles for modeling catalyst deactivation, are (i) to describe the mechanisms that take place and model them accordingly, and (ii) to model the catalyst activity and how it changes with the catalyst age, combined with a population/residence time balance (RTD) for the catalyst. Usually, both the activity of the catalyst and the RTD of the reactor are found experimentally. The idea of activity and population balance is relatively mature (Danckwerts, 1953; Hulbert and Katz, 1964; Himmelblau and Bischoff, 1968), and it is usually assumed that the catalyst age distribution is in steady-state (Franks, 1972; Petersen and Pacheco, 1984; Sarkar and Gupta, 1992, 1993). However, much less information is available on its use under unsteady-state conditions (Fernández-Sempere et al., 1995).

The aim of this study is to gain insight into control relevant consequences of common assumptions used in activity-based models of catalyst deactivation. The article is organized as follows. In Section 2, a comparison is given on the two principles of describing the dynamics of catalyst deactivation, and the consequences of various assumptions are discussed. In Section 3, the models are analyzed with regards to time response, time constants, and controller synthesis. Finally, the results are summarized, and some conclusions are given.

$\dagger$ Department of Process Automation, Telemark College, N-3914 Porsgrunn, Norway. 78712.

$\ddagger$ Department of Chemical Engineering, The University of Texas at Austin, Austin, TX

Reprinted with permission from Ind. Eng. Chem. Res. 2000, 39, 1242-1248. Copyright 2000 American Chemical Society. 


\section{Descriptions of catalyst deactivation}

\subsection{The example process}

Consider a process where component $\mathrm{A}$ is moved by an inert transport material into the reactor at a molar flow rate $W_{\mathrm{A}, \mathrm{i}}$. The molar content of component $\mathrm{A}$ in the tank is denoted $n_{\mathrm{A}}$. The molar efflux of component $\mathrm{A}$ from the reactor is denoted $W_{\mathrm{A}, \mathrm{e}}$. Traditionally, catalysts are described as speeding up the reaction without being consumed, which can be expressed as $\mathrm{A}+\mathrm{K} \rightarrow \mathrm{B}+\mathrm{K}$. The molar rate of generation of component A through the catalyzed reaction is denoted $W_{\mathrm{A}, \mathrm{r}}$; here $W_{\mathrm{A}, \mathrm{r}} \leqslant 0$.

In addition to component $\mathrm{A}$ (and the transport carrier), a catalyst $\mathrm{K}$ flows into the reactor with mass flow rate $w_{\mathrm{K}, \mathrm{i}}$. The catalyst in the reactor consists of active catalyst $\mathrm{K}_{\mathrm{a}}$ and deactivated catalyst $\mathrm{K}_{\mathrm{d}}$, with accumulated masses $m_{\mathrm{K}_{\mathrm{a}}}$ and $m_{\mathrm{K}_{d}}$, respectively. The total mass of accumulated catalyst is denoted $m_{\mathrm{K}}=m_{\mathrm{Ka}_{\mathrm{a}}}+m_{\mathrm{K}_{\mathrm{d}}}$. The catalyst entering the reactor through $w_{\mathrm{K}, \mathrm{i}}$ is assumed to be active, and as long as the catalyst resides in the reactor, it is transformed into deactivated catalyst: $K_{\mathrm{a}} \rightarrow K_{\mathrm{d}}$. The mass based effluxes of the catalysts are $w_{\mathrm{K}_{\mathrm{a}, \mathrm{e}}}, w_{\mathrm{K}_{\mathrm{d}, \mathrm{e}}}$, and $w_{\mathrm{K}, \mathrm{e}}=w_{\mathrm{K}_{\mathrm{a}, \mathrm{e}}}+w_{\mathrm{K}_{\mathrm{d}, \mathrm{e}}}$. The mass based rate of production of active catalyst is denoted $w_{\mathrm{K}_{\mathrm{a}}, \mathrm{r}}$, with $w_{\mathrm{K}_{\mathrm{a}}, \mathrm{r}} \leqslant 0$.

For this process, assume that we are not interested in product $\mathrm{B}$ per se; this may be the case when, e.g., A is a pollutant that is reduced to an unharmful product $\mathrm{B}$.

\subsection{Deactivation mechanism model}

Material balances for components $A$ and $K_{\mathrm{a}}$ are expressed by

$$
\begin{aligned}
\frac{\mathrm{d} n_{\mathrm{A}}}{\mathrm{d} t} & =W_{\mathrm{A}, \mathrm{i}}-W_{\mathrm{A} . \mathrm{e}}+W_{\mathrm{A}, \mathrm{r}} \\
\frac{d m_{\mathrm{K}, \mathrm{a}}}{\mathrm{d} t} & =w_{\mathrm{K}_{\mathrm{a}}, \mathrm{i}}-w_{\mathrm{K}_{\mathrm{a}}, \mathrm{e}}+w_{\mathrm{K}_{\mathrm{a}}}, r
\end{aligned}
$$

Here, the influxes will typically be given by upstream conditions. The effluxes will be given by the hydraulic flow through the reactor, while the reaction rates will be given by the reaction mechanisms that take place.

The ideas used in this article can be applied to any flow pattern. To illustrate the ideas, we choose to apply them to a well mixed reactor. In that case, the effluxes are given by

$$
\begin{aligned}
& W_{\mathrm{A}, \mathrm{e}}=q_{\mathrm{e}} \frac{n_{\mathrm{A}}}{V} \\
& w_{\mathrm{K}_{\mathrm{a}, \mathrm{e}}}=q_{\mathrm{e}} \frac{m_{\mathrm{K}_{\mathrm{e}}}}{V}
\end{aligned}
$$

where $q_{\mathrm{e}}$ is the volumetric efflux from the reactor, and $V$ is the reactor volume.

If the reactions $A+K_{a} \rightarrow B+K_{a}$ and $K_{a} \rightarrow K_{d}$ are elementary reactions (Levenspiel, 1972; Laidler, 1987), the rates of generation of $A$ and $K_{a}$ are

$$
\begin{aligned}
& W_{\mathrm{A}, \mathrm{r}}=-k_{0} \frac{n_{\mathrm{A}}}{V} m_{\mathrm{K}_{\mathrm{a}}} \\
& w_{\mathrm{K}_{\mathrm{a}}, \mathrm{r}}=-k_{\mathrm{d}} m_{\mathrm{K}_{\mathrm{a}}}
\end{aligned}
$$


and the complete continuous stirred tank reactor (CSTR) model, with $w_{K_{a}, \mathrm{i}}=w_{\mathrm{K}, \mathrm{i}}$, becomes

$$
\begin{aligned}
\frac{\mathrm{d} n_{\mathrm{A}}}{\mathrm{d} t} & =W_{\mathrm{A}, \mathrm{i}}-q_{\mathrm{e}} \frac{n_{\mathrm{A}}}{V}-k_{0} \frac{n_{\mathrm{A}}}{V} m_{\mathrm{K}_{\mathrm{a}}} \\
\frac{\mathrm{d} m_{\mathrm{K}_{\mathrm{a}}}}{\mathrm{d} t} & =w_{\mathrm{K}, \mathrm{i}}-q_{\mathrm{e}} \frac{m_{\mathrm{K}_{\mathrm{a}}}}{V}-k_{\mathrm{d}} m_{\mathrm{K}_{\mathrm{a}}}
\end{aligned}
$$

In practice, heterogeneous catalysts are rarely either active or deactivated; usually they exhibit a site activity distribution, e.g. (Zucchini and Cecchin, 1983). In this case, it may be necessary to formulate a mass balance for every site activity. Good model predictions can, however, still be achieved assuming a single-site activity, e.g. in modern polymerization reactors with homogeneous catalysts (Soares and Hamielec, 1996).

\subsection{Activity based model}

Often, it is convenient to not directly distinguish between active catalyst $\left(\mathrm{K}_{\mathrm{a}}\right)$ and deactivated catalyst $\left(\mathrm{K}_{\mathrm{d}}\right)$; instead the catalyst is considered as a single component $\mathrm{K}$ with accumulated mass $m_{\mathrm{K}}$ where a part of it, $\bar{m}_{\mathrm{K}}$, participates in the reaction $\mathrm{A}+\mathrm{K} \rightarrow \mathrm{B}+\mathrm{K}$. The concept of activity is used to describe to what degree the catalyst actually is active or deactivated. The fraction of $m_{\mathrm{K}}$ participating in the reaction is called the (average) activity $\bar{a}$ :

$$
\bar{a} \triangleq \bar{m}_{\mathrm{K}} / m_{\mathrm{K}}
$$

The activity varies with the age $\alpha$ of the catalyst. Let $\mu_{\mathrm{K}}$ denote the age distribution of total catalyst mass $m_{\mathrm{K}}$ such that

$$
m_{\mathrm{K}}=\int_{0}^{\infty} \mu_{\mathrm{K}}(\alpha) \mathrm{d} \alpha
$$

and where $\lim _{\alpha \rightarrow \overline{0}} \mu_{\mathrm{K}}=0$ denotes fresh catalyst that has still not been deactivated, while $\lim _{x \rightarrow \infty} \mu_{\mathrm{K}}$ is the catalyst fraction of infinite age-which should be zero. Then the activity distribution $a$ of a catalyst at age $\alpha$ is defined as $a(\alpha) \triangleq \mathrm{d} \bar{m}_{\mathrm{K}} / \mathrm{d} m_{\mathrm{K}}$, which yields

$$
m_{\mathrm{K}}=\int_{0}^{\infty} a(\alpha) \mu_{\mathrm{K}}(\alpha) \mathrm{d} \alpha
$$

In order to describe the catalyzed reaction using activity, the mole balance for component $\mathrm{A}$ is unchanged, (equation (1)). However, this time, the reaction rate $W_{\mathrm{A}}$, in equation (1) depends on $n_{\mathrm{A}}$ and the amount $\bar{m}_{\mathrm{K}}$ of active catalyst.

In practice, the activity distribution $a(\alpha)$ and the age distribution $\mu_{\mathrm{K}}(\alpha)$ are often found experimentally, and $\bar{m}_{\mathrm{K}}$ is given by equation (7). However it is also possible to compute $a(\alpha)$ and $\mu_{\mathrm{K}}(\alpha)$ theoretically from deactivation mechanisms and flow patterns in the reactor. For the analysis in this paper, the latter approach is chosen.

First we find the age distribution. The population balance for catalyst mass in the reactor at age $\alpha$ is:

$$
\frac{\mathrm{d} \mu_{\mathrm{K}}}{\mathrm{d} t}=\hat{w}_{\mathrm{K}, \mathrm{i}}-\hat{w}_{\mathbf{K}, \mathbf{e}}
$$


where $\hat{w}_{\mathrm{K}, \mathrm{i}}$ and $\hat{w}_{\mathrm{K}, \mathrm{c}}$ are influx and efflux rates of catalyst $\mathrm{K}$ at age $\alpha$, respectively, defined by $\int_{0}^{\infty} \hat{w}_{\mathrm{K}, \mathrm{i}} \mathrm{d} \alpha=w_{\mathrm{K}, \mathrm{i}}$ and $\int_{0}^{\infty} \hat{w}_{\mathrm{K}, \mathrm{e}} \mathrm{d} \alpha=w_{\mathrm{K}, \mathrm{e}}$. Furthermore, when only fresh catalyst is assumed to enter the reactor,

$$
\hat{w}_{\mathrm{K}, \mathbf{i}}=\delta(\alpha) w_{\mathbf{K}, \mathbf{i}}
$$

where $\delta(\alpha)$ is Dirac's $\delta$ distribution. Also, since $\mu_{\mathrm{K}}$ is a function of both time and age,

$$
\frac{\mathrm{d} \mu_{\mathrm{K}}(t, \alpha)}{\mathrm{d} t}=\frac{\partial \mu_{\mathrm{K}}}{\partial t} \frac{\mathrm{d} t}{\mathrm{~d} t}+\frac{\partial \mu_{\mathrm{K}}}{\partial \alpha} \frac{\mathrm{d} \alpha}{\mathrm{d} t}
$$

where the speed of aging by definition is unity, $\mathrm{d} \alpha / \mathrm{d} t=1$. Thus, the population balance becomes

$$
\frac{\partial \mu_{\mathrm{K}}}{\partial t}+\frac{\partial \mu_{\mathrm{K}}}{\partial \alpha}=\delta(\alpha) w_{\mathrm{K}, \mathrm{i}}-\hat{w}_{\mathrm{K}, \mathrm{e}}
$$

The boundary condition for this equation is

$$
\lim _{\alpha \rightarrow 0^{-}} \mu(t, \alpha)=0
$$

which means that there can not be catalyst with negative age.

In the case of a well mixed reactor, $W_{\mathrm{A}, \mathrm{e}}$ is given by equation (3). For the population balance in equation (9),

$$
\hat{w}_{\mathrm{K}, \mathrm{e}}=q_{\mathrm{e}} \frac{\mu_{\mathrm{K}}}{V}
$$

If in addition the reaction rate $\mathrm{A}+\mathrm{K} \rightarrow \mathrm{B}+\mathrm{K}$ is an elementary reaction, then

$$
W_{\mathrm{A}, \mathrm{r}}=-k_{0} \frac{n_{\mathrm{A}}}{V} \bar{m}_{\mathrm{K}}
$$

and the material balance for $\mathrm{A}$, equation (1), becomes

$$
\frac{\mathrm{d} n_{\mathrm{A}}}{\mathrm{d} t}=W_{\mathrm{A}, i}-q_{\mathrm{e}} \frac{n_{\mathrm{A}}}{V}-k_{0} \frac{n_{\mathrm{A}}}{V} \bar{m}_{\mathrm{K}}
$$

Next, we find the activity distribution $a(\alpha)$. If the deactivation follows $\mathrm{K}_{\mathrm{a}} \rightarrow \mathrm{K}_{\mathrm{d}}$ as an elementary reaction in a CSTR, the activity distribution will be

$$
a(\alpha)=\mathrm{e}^{-k_{\mathrm{d}} \alpha}
$$

which follows from equation (6) modified to be a batch reactor, where time develops with the same speed as age.

The model of the CSTR with elementary reactions now consists of equations. (7), (9), (10), (11), (12) and (13) .

If the deactivation is more complicated than the elementary reaction $\mathrm{K}_{\mathrm{a}} \rightarrow \mathrm{K}_{\mathrm{d}}$ and/ or if the reactor is not well mixed, then a different age distribution $a(\alpha)$ will be found experimentally.

\subsection{Overview of models and common assumptions}

Model 1: Mechanistic description To illustrate the two modeling principles and prepare models for analysis of dynamic properties, we assume that the reactor is well mixed, and that the reactions that take place are elementary reactions.

Model 1 is based upon the mechanistic description given by equations (5) and (6). 
Model 2: Activity and full population balance The full population balance is given by equation (9). It is quite common to normalize $\mu_{\mathrm{K}}(\alpha)$ and $\hat{w}_{\mathrm{K}, \mathrm{e}}(\alpha)$ to $I(\alpha)=\mu_{\mathrm{K}}(\alpha) / m_{\mathrm{K}}$ and $E(\alpha)=\hat{w}_{\mathrm{K}, \mathrm{e}}(\alpha) / \mathrm{w}_{\mathrm{K}, \mathrm{e}}$, respectively, where $\int_{0}^{\infty} I(\alpha) \mathrm{d} \alpha=1$ and $\int_{0}^{\infty} E(\alpha) \mathrm{d} \alpha=1$. Here, $I(\alpha)$ is the internal-age distribution of the reactor while $E(\alpha)$ is the exit-age distribution of the reactor (or the residence time distribution, RTD). When these expressions for $\mu_{\mathrm{K}}$ and $\hat{w}_{\mathrm{K}, \mathrm{e}}$ are inserted into equation (9), the full population balance leads to

$$
\frac{\partial I}{\partial t}+\frac{\partial I}{\partial \alpha}=[\delta(\alpha)-I] \frac{w_{\mathrm{K}, \mathrm{i}}}{m_{\mathrm{K}}}-(E-I) \frac{w_{\mathrm{K}, \mathrm{e}}}{m_{\mathrm{K}}}
$$

In the case of a well mixed reactor, the population balance is

$$
\frac{\partial \mu_{\mathrm{K}}}{\partial \mathrm{t}}+\frac{\partial \mu_{\mathrm{K}}}{\partial \alpha}=\delta(\alpha) w_{\mathrm{K}, \mathrm{i}}-q_{\mathrm{e}} \frac{\mu_{\mathrm{K}}}{V}
$$

If we choose to use a normalized population balance, assuming well mixedness leads to $E(\alpha)=I(\alpha)$ and

$$
\frac{\partial I}{\partial t}+\frac{\partial I}{\partial \alpha}=[\delta(\alpha)-I] \frac{w_{\mathrm{K}, \mathrm{i}}}{m_{\mathrm{K}}}
$$

where $\mu_{\mathrm{K}}(\alpha)$ is found as discussed above. Himmelblau and Bischoff (1968) developed this normalized population balance directly.

In the sequel, it is useful to introduce the hydraulic time constant $\theta$, which we define to be

$$
\theta \triangleq \frac{V}{q_{\mathrm{e}}}=\frac{m_{\mathrm{K}}}{w_{\mathrm{K}, \mathbf{i}}}
$$

When $\theta$ is a constant with respect to time, Laplace transforming equation (15) with respect to time leads to an ordinary differential equation (ODE) in $\alpha$ (e.g., ref 15), which can be solved to yield $\mu_{\mathrm{K}}(\alpha ; s)$. Next, $\bar{m}_{\mathrm{K}}(s)$ is found from equations (7) and (13). Finally, inverse Laplace transforming the resulting expression $\bar{m}_{\mathrm{K}}(s)$ yields

$$
\frac{d \bar{m}_{\mathrm{K}}}{\mathrm{d} t}=w_{\mathrm{K}, \mathrm{i}}-\left(k_{\mathrm{d}}+1 / \theta\right) \bar{m}_{\mathrm{K}}
$$

which together with

$$
\frac{\mathrm{d} n_{\mathrm{A}}}{\mathrm{d} t}=W_{\mathrm{A}, \mathrm{i}}-\left(k_{0} \frac{\bar{m}_{\mathrm{K}}}{V}+1 / \theta\right) n_{\mathrm{A}}
$$

constitutes model 2. Note that models 1 and 2 are equivalent except for the notation for the active catalyst mass, hence these models are collectively referred to as models $1-2$.

Model 3: Activity and steady-state catalyst mass In their classic article, Hulburt and Katz (1964) developed a description of how the RTD evolves to its steady-state value. On the basis of a population balance, they found that the number of particles of age $\alpha$, denoted $f(t, \alpha)$, must satisfy the following equation (using our notation):

$$
\frac{\partial f}{\partial t}+\frac{\partial f}{\partial \alpha}=\frac{1}{\theta}[N \delta(\alpha)-f]
$$


where $\int_{0}^{\infty} f \mathrm{~d} \alpha=N$. Utilizing $M_{\mathrm{K}} f / N_{\mathrm{A}}=\mu_{\mathrm{K}}$ where $M_{\mathrm{K}}$ is the molar mass of the catalyst and $N_{\mathrm{A}}$ is Avogradro's number, the model in equation (20) can be changed to describe $\mu_{\mathrm{K}}$ :

$$
\frac{\partial \mu_{\mathrm{K}}}{\partial t}+\frac{\partial \mu_{\mathrm{K}}}{\partial \alpha}=\frac{1}{\theta}\left[m_{\mathrm{K}} \delta(\alpha)-\mu_{\mathrm{K}}\right]
$$

which differs from equation (15). The reason for this discrepancy is that Hulburt and Katz assumed a 'fixed inventory of $N$ particles', in other words that the accumulated mass of catalyst in $V$ is in steady-state. With this assumption and assuming a CSTR, we find $w_{\mathrm{K}, \mathrm{i}}=q_{\mathrm{e}} m_{\mathrm{K}} / V=m_{\mathrm{K}} / \theta$, which relates Hulburt and Katz's model to equation (15).

With constant hydraulic time constant $\theta$, using the Laplace transform on equation (21) leads to

$$
\frac{d \bar{m}_{\mathrm{K}}}{\mathrm{d} t}=\frac{1}{\theta} m_{\mathrm{K}}-\left(k_{\mathrm{d}}+1 / \theta\right) \bar{m}_{\mathrm{K}}
$$

Assuming a CSTR, mass balance for $m_{\mathrm{K}}$ becomes

$$
\frac{\mathrm{d} m_{\mathrm{K}}}{\mathrm{d} t}=w_{\mathrm{K}, \mathrm{i}}-\frac{1}{\theta} m_{\mathrm{K}}
$$

We let model 3 consist of equations (19), (22) and (23).

Because Hulburt and Katz assumed $m_{\mathrm{K}}=$ constant $\Rightarrow m_{\mathrm{K}} / \theta=\mathrm{w}_{\mathrm{K}, \mathrm{i}}$, model 3 can actually be reduced to model 2 . For comparison, we prefer to still keep the somewhat artificial model 3.

Model 4: Activity and steady-state RTD In introductory reaction engineering books (Levenspiel, 1972; Fogler, 1992), it is assumed that the age distribution is in steadystate, i.e., $\partial I / \partial t=0$, or $\partial \mu_{\mathrm{K}} / \partial t=0$ in equation (15). This assumption leads, via Laplace transformation, to the following model for $\vec{m}_{\mathbf{K}}$ :

$$
\bar{m}_{\mathrm{K}}=\frac{m_{\mathrm{K}} / \theta}{k_{\mathrm{d}}}+1 / \theta
$$

which inserted into equation (19) gives

$$
\frac{\mathrm{d} n_{\mathrm{A}}}{\mathrm{d} t}=W_{\mathrm{A}, \mathrm{i}}-\left(\frac{k_{0}}{V} \frac{m_{\mathrm{K}} / \theta}{k_{\mathrm{d}}+1 / \theta}+1 / \theta\right) n_{\mathrm{A}}
$$

Model 4 thus consists of equations (23) and (25).

Model 5: Activity with both catalyst mass and RTD in steady-state If we add the assumption that $m_{\mathrm{K}}$ is a constant in model 4 , then the total model reduces to

$$
\frac{\mathrm{d} n_{\mathrm{A}}}{\mathrm{d} t}=W_{\mathrm{A}, \mathrm{i}}-\left(\frac{k_{0}}{V} \frac{w_{\mathrm{K}, \mathrm{i}}}{k_{\mathrm{d}}+1 / \theta}+1 / \theta\right) n_{\mathrm{A}}
$$

which we name model 5 .

\section{Analysis of the models}

\subsection{Comparison of time responses}

Based upon the assumption that $V / q=$ constant $=\theta$, we developed five finite dimensional dynamic models for the CSTR system with catalyst deactivation in section 2 . 
Table 1. Parameters and initial values $(t=0)$ used in the simulations.

\begin{tabular}{|c|c|c|c|}
\hline Parameter & Value & Dimension & Comment \\
\hline$\theta=V / q$ & 1 & time & hydraulic time constant, time invariant \\
\hline $\boldsymbol{V}$ & 1 & volume & reactor volume \\
\hline $\begin{array}{l}k_{\mathrm{d}} \\
\tau\end{array}$ & $\begin{array}{l}\text { see } \tau \\
\theta /\left(1 / k_{\mathrm{d}}\right)\end{array}$ & 1/time & $\begin{array}{l}1 / k_{\mathrm{d}}=\text { catalyst deactivation time constant } \\
\text { dimensionless; } \tau \in\{0.1,1,10,100\}\end{array}$ \\
\hline$k_{0}$ & 1 & $1 /$ (time $\cdot$ mass) & preexponential factor for reaction $\mathrm{A}+\mathrm{K} \rightarrow \mathrm{B}+\mathrm{K}$ \\
\hline$w_{\mathbf{K}, \mathbf{i}}$ & $1-2$ & mass/time & unit step from 1 to 2 at $t=1$ \\
\hline$W_{\mathrm{A}, \mathrm{i}}$ & 1 & $\mathrm{~mol} /$ time & constant in the simulations \\
\hline$m_{\mathrm{K}}$ & ss & mass & in the steady state: $m_{\mathbf{K}}=w_{\mathrm{K}, \mathrm{i}} \theta$ \\
\hline $\bar{m}_{\mathbf{K}}$ & ss & mass & in the steady state: $\bar{m}_{\mathbf{K}}=m_{\mathbf{K}} /(1+\tau)$ \\
\hline$m_{\mathbf{K}, \mathbf{a}}$ & ss & mass & in the steady state: $m_{\mathbf{K}, \mathbf{a}}=\bar{m}_{\mathbf{K}}$ \\
\hline$n_{\mathrm{A}}$ & ss & mol & in the steady state: $\left.n_{\mathrm{A}}=W_{\mathrm{A}, \mathrm{i}} / k_{0} \bar{m}_{\mathrm{K}} / V+1 / \theta\right)$ \\
\hline
\end{tabular}

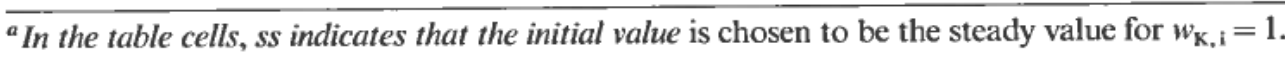

Models 1, 2, and 4 are of order 2, model 3 is of order 3, and model 5 is of order 1. Thus, not all of the models can simultaneously be equivalent. Although model 1 and model 2 appear to be different, they are in fact identical; $m_{\mathrm{K}_{\mathrm{a}}}$ and $\bar{m}_{\mathrm{K}}$ play identical roles.

Models 1-5 can be compared via simulation to give a qualitative picture of the differences among the models. In doing so, it is useful to introduce the dimensionless ratio $\tau$ between the hydraulic time constant $\theta$ and the time constant for catalyst deactivation, $1 / k_{\mathrm{d}}$ :

$$
\tau \triangleq \frac{\theta}{1 / k_{\mathrm{d}}}=k_{\mathrm{d}} \theta
$$

This ratio $(\tau)$ is the Damköhler number for the catalyst deactivation. When $\tau$ is small, the catalyst deactivates slowly compared to the hydraulic time constant (the common case). When $\tau$ is large, the catalyst deactivates relatively fast.

The simulations show the time evolution of $n_{\mathrm{A}}$ resulting from a step change in the catalyst feed rate $w_{\mathrm{K}, \mathrm{i}}$. Table 1 lists the parameters, initial values, etc., which were used in the simulations (ss $=$ steady-state). Figure 1 shows the responses $n_{\mathrm{A}}(t)$ for each of the five models.

For the case in which $\tau=0.1$, the responses of models 1-2 and model 4 in Figure 1 are virtually indistinguishable. When $\tau=100 \gg 1$, the responses of models $1-2$ and model 5 are indistinguishable; the same holds true for model 3 and model 4 . These results indicate that in the desired case of a relatively slow catalyst deactivation, the common assumption of steady-state RTD is relatively good; i.e. in the case of time response, models $1-2 \approx$ model 4 when $\tau$ is 'small'. However, when $\tau=1$, there is a marked difference between models $1-2$ and model 4 .

\subsection{Comparison of eigenvalues}

The dynamic characteristics of the models can be compared using the eigenvalues of the linearized models. Let $A_{\mathrm{j}}$ denote the Jacobian of the vector field of model $j$, with respect to its state. The eigenvalues of $A_{\mathrm{j}}$ are displayed in Table 2. It is of particular interest to study the eigenvalues as $\tau$ is small or large; these eigenvalues are also displayed in Table 2.

Table 2 shows that three distinct eigenvalues occur in the various models. When $\tau$ is small ( $\tau \rightarrow 0$, the desired case), these three eigenvalues collapse to two different 

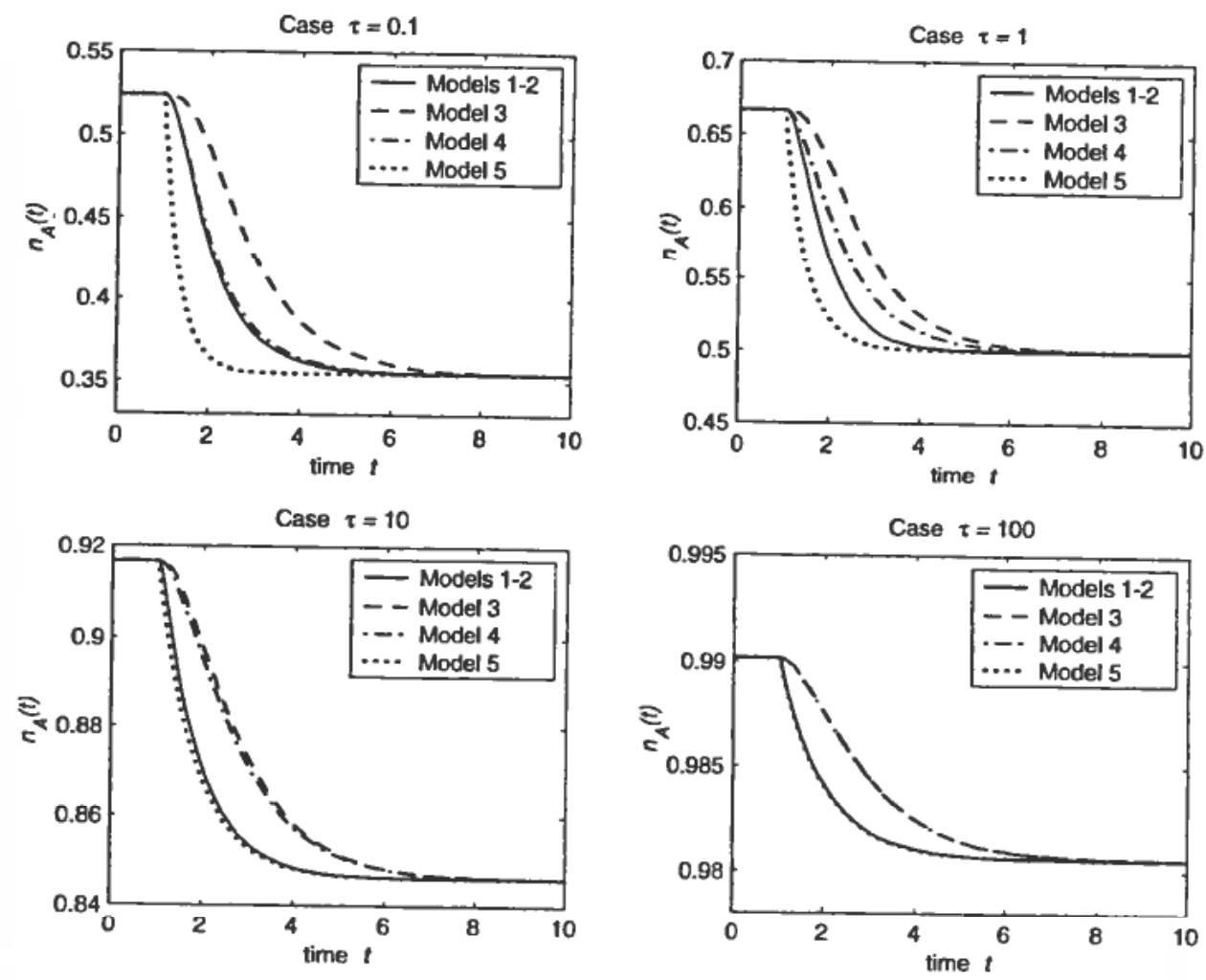

Figure 1. Number of moles $n_{\mathrm{A}}$ as a function of time for $\tau \in\{0.1,1,10,100\}$.

Table 2. Eigenvalues for the Jacobians $\left(A_{j}\right)$ of the Models $(j)$ at Steady State ${ }^{a}$

\begin{tabular}{lcccccc}
\hline eigenvalue $\lambda$ & $\lim _{\tau \rightarrow 0} \lambda$ & $\lim _{\tau \rightarrow 0} \lambda$ & $A_{1-2}$ & $A_{3}$ & $A_{4}$ & $A_{5}$ \\
\hline$-\left(\frac{k_{0}}{V} \frac{0}{1+\tau} w_{\mathrm{K}, \mathrm{i}}+1 / \theta\right)$ & $-\left(\frac{k_{0}}{V} 0 w_{\mathrm{K}, \mathrm{i}}+1 / \theta\right)$ & $-1 / \theta$ & $\mathrm{x}$ & $\mathrm{x}$ & $\mathrm{x}$ & $\mathrm{x}$ \\
$-(1+\tau) / 0$ & $-1 / 0$ & $-\infty$ & $\mathrm{x}$ & $\mathrm{x}$ & & \\
$-1 / \theta$ & $-1 / 0$ & $-1 / 0$ & & $\mathrm{x}$ & $\mathrm{x}$ \\
\hline
\end{tabular}

${ }^{a}$ If a specific eigenvalue belongs to $A_{j}$, this is marked by x.

eigenvalues, while when $\tau$ is large $(\tau \rightarrow \infty)$, a single eigenvalue dominates (the eigenvalue at $-\infty$ indicates a mode that reaches steady-state infinitely fast).

When $\tau$ is small, models $1-2$ and model 4 share dominating eigenvalues, while model 5 has one eigenvalue and model 3 three eigenvalues. This is consistent with the simulations for $\tau=0.1$ in Figure 1. When $\tau$ is large, models $1-2$ and model 5 share eigenvalues, while models 3 and 4 share eigenvalues. This is consistent with the simulations for $\tau=100$ in Figure 1 .

As Table 2 shows, the assumption of steady-state RTD applied in model 4, introduces a change in one of the eigenvalues of models $1-2$, while the other eigenvalue is unchanged: Eigenvalue $\lambda_{1-2}=-(1+\tau) / \theta$ is changed into $\lambda_{4}=-1 / \theta$. It is of interest 

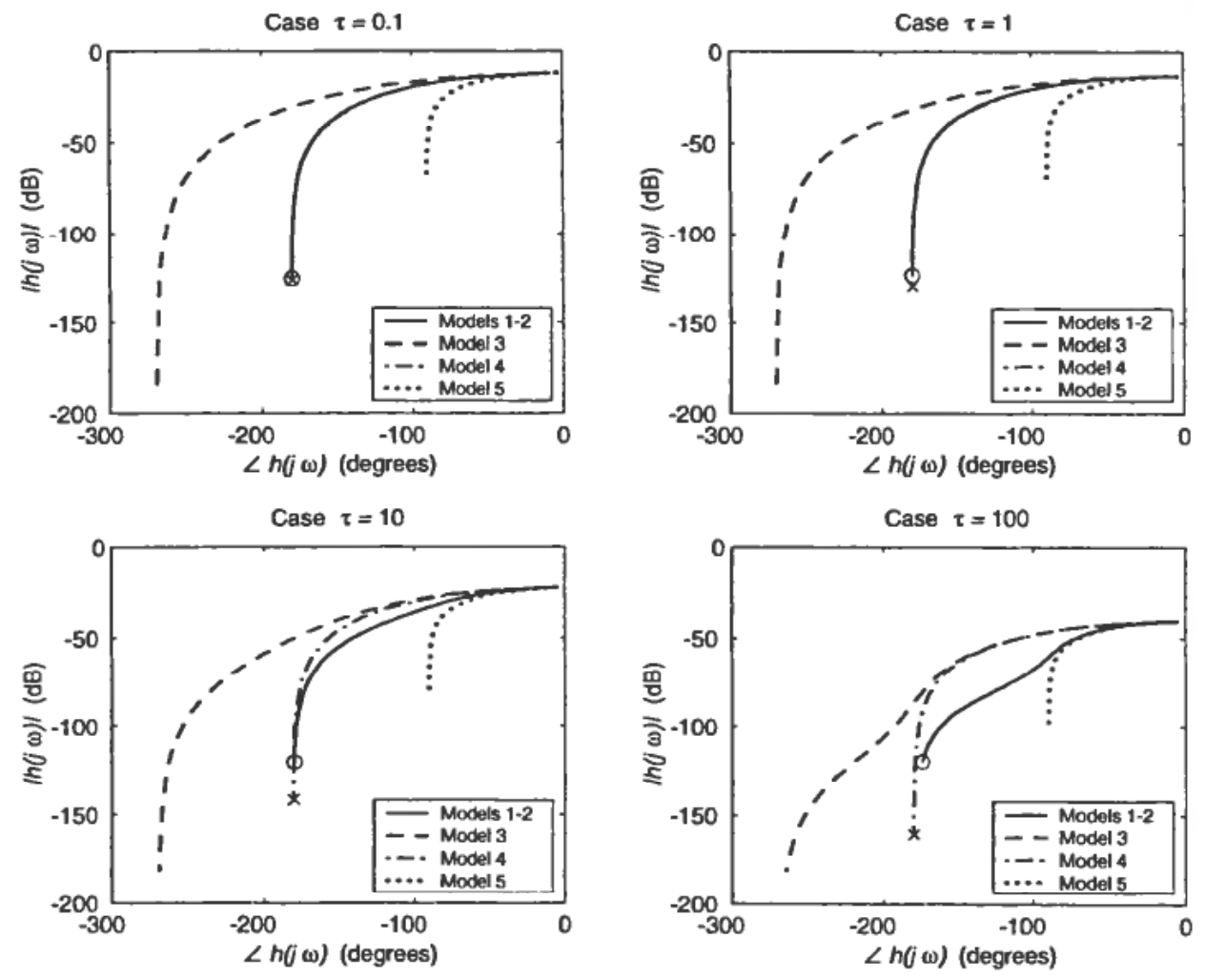

Figure 2. Polar plots of transfer functions $n_{\mathrm{A}}(s) /-w_{\mathrm{K}, i}(s)$ for $\tau \in\{0.1,1,10,100\}$, using frequency $\omega \in\left[10^{-1}, 10^{3}\right]$ as a parameter. For $\omega=10^{3}$, the phase-amplitude pair for models $1-2$ is marked with $O$, while for model 4 the pair is marked with $\times$.

to have a measure of the relative change in the dynamics caused by the steady-state RTD assumption; we choose to measure the relative change $\varepsilon_{\mathrm{T}}$ in time constant $|1 / \lambda|$ :

$$
\varepsilon_{\mathrm{T}} \triangleq\left|\frac{\left|1 / \lambda_{1-2}\right|-\left|1 / \lambda_{4}\right|}{\left|1 / \lambda_{1-2}\right|}\right|=\tau
$$

Hence, for $\tau=0.1$, the relative change $\varepsilon_{\mathrm{T}}$ is $10 \%$, while when $\tau=1.0, \varepsilon_{\mathrm{T}}$ is $100 \%$.

\subsection{Comparison of transfer functions}

It is of interest to compare the models with respect to controller synthesis. The four different models are linearized around their respective operating points at $t=0$ as listed in Table 1, yielding a linear description of form $\dot{x}=\mathbf{A} x+\mathbf{B} u, y=\mathbf{C} x$, where $u$ is the deviation of $w_{\mathrm{K}, \mathrm{i}}$ from its steady-state value at $t=0$, and $y$ is the deviation of $n_{\mathrm{A}}$ from its steady-state value at $t=0$. Matrix A equals the Jacobians $A_{\mathrm{j}}$ discussed in section 3.2. Because the steady-state gain from $w_{\mathrm{K}, \mathrm{i}}$ to $n_{\mathrm{A}}$ is negative, we study transfer functions $h(s)=-\mathbf{C}(s I-\mathbf{A})^{-1} \mathbf{B}$. With $s=j \omega(j \triangleq \sqrt{-1})$ and parameter $\omega \in\left[10^{-1}, 10^{-3}\right]$, the amplitude of $h(s)$ (in decibels) can be plotted as a function of the phase of $h(s)$ (in degrees). The results are shown in Figure 2 for the cases in which $\tau \in\{0.1,1,10,100\}$.

As seen from Figure 2, at high frequencies the phases of the models approach 
$-90 \times$ model order. Although the polar plots of the transfer functions for models 1-2 and model 4 are almost indistinguishable at $\tau=1$, there is a marked difference in their time responses as indicated in Figure 1. While the time responses for models 3 and 4 appear to be almost identical at $\tau=100$ in Figure 1, their transfer functions are equal only at low frequencies.

Figure 2 shows that the shape of the polar plots of models 1-2 and model 4 are almost indistinguishable unless $\tau$ becomes relatively large $(\tau=10)$; to distinguish them their phase-amplitude pair at $\omega=10^{3}$ are marked with $O$ (models 1-2) and $\times$ (model 4). From a control point of view, it is the shape of the polar plot that determines the stability of the controlled system. This has the important consequence that assuming steady-state RTD may have relatively little impact on the stability margins of a controlled system for manipulating $n_{\mathrm{A}}$ via $w_{\mathrm{K}, \mathrm{i}}$. What will be changed, though, is the bandwidth of the closed loop system: this is seen by the way the polar plot for model 4 is stretched relative to the polar plot for models $1-2$ when $\tau$ increases, see the point on the polar curves where $\omega=10^{3}$ in Figure 2.

\section{Discussion and conclusions}

We have studied two different principles for modeling catalyst deactivation. Several simplifying assumptions are common to all models in this study, and it is relatively straightforward to relax most of them. To remove the CSTR assumption, it is necessary to introduce a distributed parameter model of the flow pattern. If the deactivation mechanism is more complicated/non-elementary, the relevant modifications must be introduced. If product from the reactor is refluxed to the inlet, it is necessary to modify the models to allow for deactivated catalyst in the feed. If a site activity distribution is important, the sites can be considered belonging to different catalysts, each with a single-site activity. The common assumptions were chosen to highlight the various aspects of additional assumptions. Five dynamic models were developed. Model 1 (second-order model) was based upon a mechanistic description of the catalyst deactivation, while models $2-5$ were based upon a catalyst activity/RTD principle with various levels of additional assumptions.

In model 2, it was assumed that the hydraulic time constant of the system was constant; the resulting model is identical to model 1. Model 3 (third-order model) was based upon the classical dynamic population balance model/RTD in Hulburt and Katz (1964) but did not take advantage of their assumption of a constant accumulated catalyst mass. If this additional assumption is applied to model 3 , then this model becomes identical to models 1 and 2 for a CSTR, which is interesting. To arrive at model 4 (second-order model), model 2 was restricted with the assumption of a steadystate RTD. Finally, in model 5 (first-order model), both constant accumulated catalyst mass and steady-state RTD were assumed.

Models 1-2 represents a relatively complete dynamic model under the common assumptions, while model 4 represents a widespread additional assumption. Thus, special emphasis is put on comparing models $1-2$ and model 4 . An important parameter in the study is the catalyst Damköhler number $\tau$, which is the ratio between the hydraulic time constant $\theta$ and the time constant of catalyst deactivation $1 / k_{\mathrm{d}}$. When $\tau$ is small $(\tau \ll 1)$, the catalyst remains active for many residence times of the reactant. This is a common case in practice, and the catalyst is often fixed within the reactor. When $\tau$ is large $(\tau \gg 1)$, the catalyst will decay in fractions of the reactant residence time. This case will only be acceptable when the catalyst is inexpensive compared to the product. 
It is shown that when $\tau \rightarrow 0$ (no catalyst deactivation), models $1-2$ and model 4 become identical. As the catalyst deactivation becomes more pronounced ( $\tau$ increases towards unity), models 1-2 and model 4 show more and more differing time responses. It is shown that the relative change $\varepsilon_{\mathrm{T}}$ in one time constant of models $1-2$ by introducing the assumptions leading to model 4 , is given by $\varepsilon_{\mathrm{T}}=\tau$. Thus, when $\tau=0.1$, the relative change in the time constant of model 4 is $\varepsilon_{\mathrm{T}}=10 \%$. With $\tau=1$, this change is $\varepsilon_{\mathrm{T}}=100 \%$.

An interesting result from the study is that introducing a steady-state RTD assumption appears to be less critical when it comes to stability margins in a feedback system: In spite of the sensitivity of $\tau$ in the time constant as discussed above, the shape of the transfer function in a polar diagram does not change much until $\tau$ is rather large. This indicates that by basing the control design on the assumptions of model 4 , the predicted stability margins of the resulting closed loop system may not be too far away from the observed stability margins with models $1-2$ being 'reality'. However, a different bandwidth will be achieved in practice, than what is predicted by assuming steadystate RTD.

The insight gained from the common and additional assumptions will carry over to more general catalyst deactivation problems: When the time constants of catalyst decay approaches other time constants in the system, it may be necessary to include the dynamics of the catalyst decay in the model. Often, a steady-state RTD is employed just like in model 4, without considering the justification of this assumption (Franks, 1972; Sarkar and Gupta, 1992, 1993).

\section{Acknowledgment}

Suggestions for improvements by reviewers are gratefully acknowledged.

\section{Nomenclature}

\section{Flow rates}

The following general nomenclature is used for flow rates: volumetric flow rate is denoted $q$, mass flow rate is denoted $w$, and molar flow rate is denoted $W$.

\section{Roman symbols}

$a, \bar{a}=$ activity of the catalyst, function of age $\alpha$ and average activity

$A_{1-2}, A_{3}, A_{4}, A_{5}=$ Jacobian of the vector field of: models 1-2 and 3-5

$\mathbf{A}, \mathbf{B}, \mathbf{C}=$ matrices in dynamic, linearized system

$c_{\mathrm{A}}, c_{\mathrm{A}, \mathrm{i}}=$ concentration of component $\mathrm{A}$ in the reactor and in the feed [mol/volume]

$E(\alpha)=$ exit-age distribution [1/time]

$f(t, \alpha)=$ number of particles of age $\alpha$, at time $t$

$h(s)=$ transfer function from $w_{\mathrm{K}, \mathrm{i}}$ to $c_{\mathrm{A}}$

$I(\alpha)=$ internal-age distribution $[1 /$ time $]$

$j=\sqrt{-1}$

$k_{\mathrm{d}}=$ rate constant: catalyst deactivation [1/time]

$k_{0}=$ rate constant: reaction $\mathrm{A}+\mathrm{K} \rightarrow \mathrm{B}+\mathrm{K}$ [volume/(mass • time)]

$\mathrm{K}, \mathrm{K}_{\mathrm{a}}, \mathrm{K}_{\mathrm{d}}=$ catalyst, active catalyst and deactivated catalyst

$m_{\mathrm{K}}, \overline{\mathrm{m}}_{\mathrm{K}}=$ accumulated mass of $\mathrm{K}$ and effective catalyst mass [mass]

$m_{\mathrm{K}_{\mathrm{a}}}, m_{\mathrm{K}_{\mathrm{d}}}=$ accumulated mass of $\mathrm{K}_{\mathrm{a}}$ and $\mathrm{K}_{\mathrm{d}}$ [mass]

$M_{\mathrm{K}}=$ molar mass of the catalyst [mass $/ \mathrm{mol}$ ] 
$n_{\mathrm{A}}, n_{\mathrm{B}}=$ no. of accumulated moles in reactor: of A and of $\mathrm{B}$ [mol]

$N_{\mathrm{A}}, N=$ Avogadro's number and number of (catalyst) particles in reactor

$q_{\mathrm{e}}=$ volumetric efflux from the reactor [volume/time]

$s=$ variable representing time for Laplace transformed quantities

$t=$ time [time]

$V=$ volume of reactor [volume]

$w_{\mathrm{K}, \mathrm{e}}, w_{\mathrm{K}, \mathrm{i}}=$ mass flow rate of catalyst: efflux and influx [mass/time]

$w_{\mathbf{K}_{\mathrm{a}}, \mathrm{e}}, w_{\mathrm{K}_{3}, \mathrm{r}}=$ mass rate of active catalyst: efflux and generation by reaction [mass/time]

$\hat{w}_{\mathrm{K}, \mathrm{i}}(\alpha), \hat{w}_{\mathrm{K}, \mathrm{e}}(\alpha)=$ mass flow rate of the catalyst of age $\alpha$ : in the output and input [mass/time ${ }^{2}$ ]

$W_{\mathrm{A}, \mathrm{e}}, W_{\mathrm{A}, \mathrm{i}}=$ molar rate of A: flow in the output and input [mol/time]

$W_{\mathrm{A}, \mathrm{r}}=$ molar rate of generation of $\mathrm{A}$ by reaction [mol/time]

$\hat{W}_{\mathrm{A}, \mathrm{r}}(\alpha)=$ molar rate of reaction of A with catalyst of age $\alpha\left[\mathrm{mol} / \mathrm{time}^{2}\right]$

\section{Greek symbols}

$\alpha=$ age in reactor [time]

$\delta(\alpha)=$ Dirac's $\delta$ function

$\varepsilon_{\mathrm{T}}=$ elative difference between changed time constants in $A_{1-2}$ and $A_{4}$

$\lambda_{1-2}, \lambda_{4}=$ changed eigenvalues of $A_{1-2}$ and $A_{4}[1 /$ time $]$

$\mu_{\mathrm{K}}(\alpha)=$ mass of catalyst that has stayed a time $\alpha$ in the reactor $\int_{0}^{\alpha} \mu_{\mathrm{K}} \mathrm{d} \alpha=m_{\mathrm{K}}$ [mass/ time]

$\theta=$ hydraulic time constant and $\theta=V I q$ when $q$ is constant [time]

$\tau=$ ratio $\theta /\left(1 / k_{\mathrm{d}}\right)=k_{\mathrm{d}} \theta$ and the catalyst Damköhler number

$\omega=$ frequency $[1 /$ time $]$

\section{Abbreviations}

CSTR $=$ continuous stirred tank reactor

$\mathrm{RTD}=$ residence time distribution

\section{REFERENCES}

FogLer, H. S. (1992). Elements of Chemical Reaction Engineering, 2nd ed; Prentice-Hall: Englewood Cliffs, New Jersey.

Butt, J. B. and Petersen E. E. (1988). Activation, Deactivation and Poisoning of Catalysts; Academic: New York.

LeVenspiel, O. (1972). Chemical Reaction Engineering, 2nd ed; John Wiley \& Sons: New York.

DANCKWERTS, P. V. (1953). Continuous flow systems. Distribution of Residence Times. Chem. Eng. Sci., Vol. 2, pp. 1-13.

Hulburt, H. M. and Katz, S. (1964). Some problems in particle technology. A statistical mechanical formulation. Chem. Eng. Sci., Vol. 19, pp. 555-574.

Himmelblau, D. M. and Bischoff, K.B. (1968). Process Analysis and Simulation: Deterministic Systems; John Wiley and Sons: New York.

Petersen, E. E. and PACHECO, M. A. (1984). Fundamental Deactivation Data from Laboratory Reactors. Chapter 19 in (Ed.) Duduković, M. P. and MrLs, P. L. Chemical and Catalytic Reactor Modeling; ACS Symposium series 237, American Chemical Society: Washington, D.C.

Franks, R. G. E. (1972). Modeling and Simulation in Chemical Engineering; John Wiley \& Sons: New York.

SARKAR, P. and Gupta, S. K. (1992). Steady State Simulation of Continuous-Flow Stirred-Tank Slurry Propylene Polymerization Reactors. Polym. Eng. Sci., Vol. 32, No. 11, pp. 732-742.

SARKar, P. and GuPta, S. K. (1993). Dynamic Simulation of Propylene Polymerization in Continuous Flow Stirred Tank Reactors. Polym. Eng. Sci., Vol. 33, No. 6, pp. 363-374. 
Fernández-Sempere, J., Font-Montesinos, R. and Espejo-Alcaraz, O. (1995). Residence Time Distribution for Unsteady-State Systems. Chemical Engineering Science, Vol. 50, No. 2, pp. 223-230.

Laidler, K. J. (1987). Chemical Kinetics, third edition; Harper \& Row: New York.

Zucchini, U. and Cecchin, G. Control of Molecular-Weight Distribution in Polyolefins Synthesized with Ziegler-Natta Catalytic Systems. Adv. Polym. Sci., 1983, Vol. 51, SpringerVerlag: Berlin.

SoARES, J. B. P. and Hamielec, A. E. (1996). Copolymerization of olefins in a series of continuous stirred-tank slurry-reactors using heterogeneous Ziegler-Natta and Metallocene catalyst. I. General dynamic mathematical model. Polymer Reaction Engineering, No. 4. (2\&3), pp. 153-192.

StePHEnSon, G. (1985). Partial Differential Equations for Scientists and Engineers, 3rd ed; Longman: London. 